\title{
Ulam-Hyers Stability and Uniqueness for Nonlinear Sequential Fractional Differential Equations Involving Integral Boundary Conditions
}

\author{
Areen Al-khateeb ${ }^{1, *(\mathbb{D})}$, Hamzeh Zureigat ${ }^{1}{ }^{\mathbb{D}}$, Osama Ala'yed $^{1}$ and Sameer Bawaneh ${ }^{2}$ \\ 1 Department of Mathematics, Faculty of Science and Information Technology, Jadara University, \\ Irbid 21110, Jordan; hamzeh.zu@jadara.edu.jo (H.Z.); alayedo@jadara.edu.jo (O.A.) \\ 2 Department of Software Engineering, Faculty of Architecture and Engineering, Rauf Denktas University, \\ Nicosia 139149, North Cyprus; Sameer.bawaneh@rdu.edu.tr \\ * Correspondence: areen.k@jadara.edu.jo; Tel.: +962-779-474-718
}

check for updates

Citation: Al-khateeb, A.; Zureigat, H.; Ala'yed, O.; Bawaneh, S. Ulam-Hyers Stability and

Uniqueness for Nonlinear Sequential Fractional Differential Equations Involving Integral Boundary Conditions. Fractal Fract. 2021, 5, 235. https://doi.org/10.3390/

fractalfract5040235

Academic Editor: John R. Graef

Received: 2 September 2021

Accepted: 6 November 2021

Published: 19 November 2021

Publisher's Note: MDPI stays neutral with regard to jurisdictional claims in published maps and institutional affiliations.

Copyright: (c) 2021 by the authors. Licensee MDPI, Basel, Switzerland. This article is an open access article distributed under the terms and conditions of the Creative Commons Attribution (CC BY) license (https:/ / creativecommons.org/licenses/by/ $4.0 /)$.

\begin{abstract}
Fractional-order boundary value problems are used to model certain phenomena in chemistry, physics, biology, and engineering. However, some of these models do not meet the existence and uniqueness required in the mainstream of mathematical processes. Therefore, in this paper, the existence, stability, and uniqueness for the solution of the coupled system of the Caputo-type sequential fractional differential equation, involving integral boundary conditions, was discussed, and investigated. Leray-Schauder's alternative was applied to derive the existence of the solution, while Banach's contraction principle was used to examine the uniqueness of the solution. Moreover, Ulam-Hyers stability of the presented system was investigated. It was found that the theoretical-related aspects (existence, uniqueness, and stability) that were examined for the governing system were satisfactory. Finally, an example was given to illustrate and examine certain related aspects.
\end{abstract}

Keywords: sequential fractional differential equation; fixed-point theorem; Ulam-Hyers stability; fractional differential equation

\section{Introduction}

In recent decades, the field of fractional-order boundary value problems has been discussed by several scientific researchers across the world. This is evident from the number of significant studies of fractional-order boundary value problems that mainly focus on extending and transforming such problems from the theoretical to the application aspect, in order to make them applicable for certain real-life phenomena. The fractional calculus essentially involves differentiation and integration to an arbitrary order, which is considered as an important tools that have facilitated many real-life phenomena in considerable fields such as physics, biology, and chemistry (see [1-4]). In addition, engineering is considered to be one of the main fields that benefits from fractional calculus, due to providing a full and comprehensive description of some complex engineering models.

Moreover, the significance of fractional calculus stretches further than scientific areas, to several other areas that influence human civilization in general. As an outcome of these efforts, many practical mathematical models that are expressed based on fractional differential equations have been developed, providing infinite description support of such mathematical models and developing a novel strategy for use in other practical fields. This leads to a new path of research that aims to have more collaboration between mathematicians and other researchers. In addition, actual practicality is considered to be one of the essential advantages of fractional-order models, such as those mentioned in the following articles [5-18]. Recently, Boutiara et al. [19] discussed the solution of a nonlinear sequential q-difference equation based on the Caputo fractional quantum derivatives, with 
nonlocal boundary value conditions containing Riemann-Liouville fractional quantum integrals at four points. The criteria and conditions of the existence and uniqueness of the solutions to the given Caputo fractional q-difference boundary value problem have been derived in this study. The stability of the proposed equation was investigated based on Ulam-Hyers stability, and the results obtained were examined by providing two examples.

The coupled system of the Caputo-type sequential fractional differential is considered as one of the most important tools available to model and simulate certain real-life phenomena. Therefore, there is a need to investigate the related theoretical aspects. Motivated by the above discussion and our review of the literature, this paper aims to discuss and analyze the following coupled system of Caputo-type sequential fractional differential equations. In particular, we aimed to investigate the existence, stability, and uniqueness of the solution to the coupled system of Caputo-type sequential fractional differential equations:

$$
\left\{\begin{array}{l}
{ }^{c} D^{\alpha-1}(D+k) x(t)=f(t, x(t), y(t)), t \in[0, T], 1<\alpha \leq 2, k>0, \\
{ }^{c} D^{\beta-1}(D+k) y(t)=g(t, x(t), y(t)), t \in[0, T], 1<\beta \leq 2, k<0,
\end{array}\right.
$$

supplemented with integral boundary conditions of the form:

$$
\left\{\begin{array}{c}
\int_{0}^{T} x(s) d s=\rho_{1} y\left(\zeta_{1}\right), \int_{0}^{T} x^{\prime}(s) d s=\rho_{2} y^{\prime}\left(\zeta_{2}\right), \\
\int_{0}^{T} y(s) d s=\mu_{1} x\left(\eta_{1}\right), \int_{0}^{T} y^{\prime}(s) d s=\mu_{2} x^{\prime}\left(\eta_{2}\right), \eta_{1}, \eta_{2}, \zeta_{1}, \zeta_{2} \in[0, T],
\end{array}\right.
$$

where ${ }^{c} D^{k}$ denote the Caputo fractional derivatives of order $k, k=\alpha, \beta$, and $f, g:[0, T] \times \mathbb{R}^{2} \rightarrow \mathbb{R}$, are given continuous functions, and $\rho_{1}, \rho_{2}, \mu_{1}, \mu_{2}$ are real constants.

\section{Preliminaries}

Firstly, we recall the definitions of fractional derivatives and integrals [1,2].

Definition 1. The Caputo derivative of fractional order $\gamma$ for $(k-1)$ - times absolutely continuous function $g:[0, \infty) \rightarrow \mathbb{R}$ is defined as:

$$
{ }^{c} D^{\gamma} g(s)=\frac{1}{\Gamma(k-\gamma)} \int_{0}^{s}(s-t)^{k-\gamma-1} g^{(m)}(t) d t, k-1<\gamma<k, k=[\gamma]+1,
$$

where $[\gamma]$ is the integer part of the real number $\gamma$.

Definition 2. The Riemann-Liouville fractional integral of order $\gamma$ for a continuous function $g$ is given by:

$$
I^{\gamma} g(t)=\frac{1}{\Gamma(\gamma)} \int_{0}^{t} \frac{g(s)}{(t-s)^{1-\gamma}} d t, \gamma>0
$$

provided that the right-hand side is point-wise defined on $[0, \infty)$.

Definition 3. Due to Miller-Ross [3], the sequential fractional derivative for a sufficiently smooth function $g(t)$ is defined as:

$$
D^{m} g(t)=D^{m_{1}} D^{m_{2}} \ldots D^{m_{n}} g(t),
$$

where $m=\left(m_{1}, m_{2}, \ldots, m_{n}\right)$ is a multi-index.

We prove the following auxiliary lemma to find the solution for the problems (1) and (2). 
Lemma 1. Let, $\phi \in C([0, T], \mathbb{R})$. Then the unique solution of the problem:

$$
\left\{\begin{array}{c}
{ }^{C} D^{\alpha-1}(D+k) x(t)=\psi(t), 1<\alpha \leq 2, \\
{ }^{C} D^{\beta-1}(D+k) y(t)=\phi(t), 1<\beta \leq 2, \\
\int_{0}^{T} x(s) d s=\rho_{1} y\left(\zeta_{1}\right), \int_{0}^{T} x^{\prime}(s) d s=\rho_{2} y^{\prime}\left(\zeta_{2}\right) \\
\int_{0}^{T} y(s) d s=\mu_{1} x\left(\eta_{1}\right), \int_{0}^{T} y^{\prime}(s) d s=\mu_{2} x^{\prime}\left(\eta_{2}\right), k>0, t \in[0, T],
\end{array}\right.
$$

is:

$$
\begin{aligned}
& x(t)=\Delta e^{-k t}\left[\mu_{2}\left(I^{\alpha-1} \psi\right)\left(\eta_{2}\right)-k \mu_{2} \int_{0}^{\eta_{2}} e^{-k\left(\eta_{2}-s\right)}\left(I^{\alpha-1} \psi\right)(s) d s\right. \\
& \left.-\int_{0}^{T}\left(I^{\beta-1} \phi\right)(s) d s+k \int_{0}^{T} \int_{0}^{x} e^{-k(x-s)}\left(I^{\beta-1} \phi\right)(s) d s d x\right] \\
& +\lambda e^{-k t}\left[\rho_{2}\left(I^{\beta-1} \phi\right)\left(\zeta_{2}\right)-k \rho_{2} \int_{0}^{\zeta_{2}} e^{-k\left(\zeta_{2}-s\right)}\left(I^{\beta-1} \phi\right)(s) d s-\int_{0}^{T}\left(I^{\alpha-1} \psi\right)(s) d s\right. \\
& \left.+k \int_{0}^{T} \int_{0}^{x} e^{-k(x-s)}\left(I^{\alpha-1} \psi\right)(s) d s d x\right] \\
& +\frac{1}{T^{2}-\mu_{1} \rho_{1}}\left[\frac{A \mu_{2}}{k}\left(I^{\alpha-1} \psi\right)\left(\eta_{2}\right)-A \mu_{2} \int_{0}^{\eta_{2}} e^{-k\left(\eta_{2}-s\right)}\left(I^{\alpha-1} \psi\right)(s) d s\right. \\
& -\frac{A}{k} \int_{0}^{T}\left(I^{\beta-1} \phi\right)(s) d s+\left(A-\rho_{1}\right) \int_{0}^{T} \int_{0}^{x} e^{-k(x-s)}\left(I^{\beta-1} \phi\right)(s) d s d x \\
& +\frac{B \rho_{2}}{k}\left(I^{\beta-1} \phi\right)\left(\zeta_{2}\right)-B \rho_{2} \int_{0}^{\zeta_{2}} e^{-k\left(\zeta_{2}-s\right)}\left(I^{\beta-1} \phi\right)(s) d s-\frac{B}{k} \int_{0}^{T}\left(I^{\alpha-1} \psi\right)(s) d s \\
& +(B-T) \int_{0}^{T} \int_{0}^{x} e^{-k(x-s)}\left(I^{\alpha-1} \psi\right)(s) d s d x+T \rho_{1} \int_{0}^{\zeta_{1}} e^{-k\left(\zeta_{1}-s\right)}\left(I^{\beta-1} \phi\right)(s) d s \\
& \left.+\mu_{1} \rho_{1} \int_{0}^{\eta_{1}} e^{-k\left(\eta_{1}-s\right)}\left(I^{\alpha-1} \psi\right)(s) d s\right]+\int_{0}^{t} e^{-k(t-s)}\left(I^{\alpha-1} \psi\right)(s) d s, \\
& \text { and: } \\
& y(t)=\theta e^{-k t}\left[\mu_{2}\left(I^{\alpha-1} \psi\right)\left(\eta_{2}\right)-k \mu_{2} \int_{0}^{\eta_{2}} e^{-k\left(\eta_{2}-s\right)}\left(I^{\alpha-1} \psi\right)(s) d s\right. \\
& \left.-\int_{0}^{T}\left(I^{\beta-1} \phi\right)(s) d s+k \int_{0}^{T} \int_{0}^{x} e^{-k(x-s)}\left(I^{\beta-1} \phi\right)(s) d s d x\right] \\
& +\tau e^{-k t}\left[\rho_{2}\left(I^{\beta-1} \phi\right)\left(\zeta_{2}\right)-k \rho_{2} \int_{0}^{\zeta_{2}} e^{-k\left(\zeta_{2}-s\right)}\left(I^{\beta-1} \phi\right)(s) d s-\int_{0}^{T}\left(I^{\alpha-1} \psi\right)(s) d s\right. \\
& \left.+k \int_{0}^{T} \int_{0}^{x} e^{-k(x-s)}\left(I^{\alpha-1} \psi\right)(s) d s d x\right] \\
& +\frac{1}{\omega}\left[\frac{C \mu_{2}}{k}\left(I^{\alpha-1} \psi\right)\left(\eta_{2}\right)-C \mu_{2} \int_{0}^{\eta_{2}} e^{-k\left(\eta_{2}-s\right)}\left(I^{\alpha-1} \psi\right)(s) d s-\frac{C}{k} \int_{0}^{T}\left(I^{\beta-1} \phi\right)(s) d s\right. \\
& +(C-T) \int_{0}^{T} \int_{0}^{x} e^{-k(x-s)}\left(I^{\beta-1} \phi\right)(s) d s d x+\frac{D \rho_{2}}{k}\left(I^{\beta-1} \phi\right)\left(\zeta_{2}\right) \\
& -D \rho_{2} \int_{0}^{\zeta_{2}} e^{-k\left(\zeta_{2}-s\right)}\left(I^{\beta-1} \phi\right)(s) d s-\frac{D}{k} \int_{0}^{T}\left(I^{\alpha-1} \psi\right)(s) d s \\
& +\left(D-\mu_{1}\right) \int_{0}^{T} \int_{0}^{x} e^{-k(x-s)}\left(I^{\alpha-1} \psi\right)(s) d s d x+\mu_{1} \rho_{1} \int_{0}^{\zeta_{1}} e^{-k\left(\zeta_{1}-s\right)}\left(I^{\beta-1} \phi\right)(s) d s \\
& \left.+T \mu_{1} \int_{0}^{\eta_{1}} e^{-k\left(\eta_{1}-s\right)}\left(I^{\alpha-1} \psi\right)(s) d s\right]+\int_{0}^{t} e^{-k(t-s)}\left(I^{\beta-1} \phi\right)(s) d s,
\end{aligned}
$$


where:

$$
\begin{aligned}
& \omega=T^{2}-\mu_{1} \rho_{1} \neq 0, \Delta=\frac{-k \rho_{2} e^{-k \zeta_{2}}}{M}, \lambda=\frac{e^{-k T}-1}{M}, \sigma=\Delta k \mu_{2} e^{-k \eta_{2}}-1 \\
& M=\left(e^{-k T}-1\right)^{2}-k^{2} \mu_{2} \rho_{2} e^{-k\left(\zeta_{2}+\eta_{2}\right)} \neq 0, \theta=\frac{-\sigma}{e^{-k T}-1}, \tau=\frac{\lambda}{e^{-k T}-1}, \\
& A=\left[\theta k T \rho_{1} e^{-k \zeta_{1}}+k \mu_{1} \rho_{1} \Delta e^{-k \eta_{1}}+\left(e^{-k T}-1\right)\left(\Delta T+\theta \rho_{1}\right)\right], \\
& B=\left[\tau k T \rho_{1} e^{-k \zeta_{1}}+k \mu_{1} \rho_{1} \lambda e^{-k \eta_{1}}+\left(e^{-k T}-1\right)\left(\lambda T+\tau \rho_{1}\right)\right], \\
& C=\left[\Delta k T \mu_{1} e^{-k \eta_{1}}+k \mu_{1} \rho_{1} \theta e^{-k \zeta_{1}}+\left(e^{-k T}-1\right)\left(\theta T+\Delta \mu_{1}\right)\right], \\
& D=\left[\lambda k T \mu_{1} e^{-k \eta_{1}}+k \mu_{1} \rho_{1} \tau e^{-k \zeta_{1}}+\left(e^{-k T}-1\right)\left(\tau T+\lambda \mu_{1}\right)\right] .
\end{aligned}
$$

Proof. The general solutions of the sequential fractional differential equations [20-23] in (3) are known as:

$$
\begin{aligned}
& x(t)=a_{0} e^{-k t}+a_{1}+\int_{0}^{t} e^{-k(t-s)}\left(I^{\alpha-1} \psi\right)(s) d s, \\
& y(t)=b_{0} e^{-k t}+b_{1}+\int_{0}^{t} e^{-k(t-s)}\left(I^{\beta-1} \phi\right)(s) d s,
\end{aligned}
$$

observe:

$$
\begin{aligned}
l x^{\prime}(t) & =-k a_{0} e^{-k t}+\left(I^{\alpha-1} \psi\right)(t)-k \int_{0}^{t} e^{-k(t-s)}\left(I^{\alpha-1} \psi\right)(s) d s, \\
y^{\prime}(t) & =-k b_{0} e^{-k t}+\left(I^{\beta-1} \phi\right)(t)-k \int_{0}^{t} e^{-k(t-s)}\left(I^{\beta-1} \phi\right)(s) d s,
\end{aligned}
$$

where $a_{i}, b_{i} \in \mathbb{R}, i=0,1$ are arbitrary constants.

Applying the conditions:

$$
\int_{0}^{T} x^{\prime}(s) d s=\rho_{2} y^{\prime}\left(\zeta_{2}\right), \int_{0}^{T} y^{\prime(s)} d s=\mu_{2} x^{\prime}\left(\eta_{2}\right) .
$$

Then we obtain:

$$
\begin{aligned}
a_{0}=\Delta\left[\mu_{2}\left(I^{\alpha-1} \psi\right)\right. & \left(\eta_{2}\right)-k \mu_{2} \int_{0}^{\eta_{2}} e^{-k\left(\eta_{2}-s\right)}\left(I^{\alpha-1} \psi\right)(s) d s \\
& \left.-\int_{0}^{T}\left(I^{\beta-1} \phi\right)(s) d s+k \int_{0}^{T} \int_{0}^{x} e^{-k(x-s)}\left(I^{\beta-1} \phi\right)(s) d s d x\right] \\
& +\lambda\left[\rho_{2}\left(I^{\beta-1} \phi\right)\left(\zeta_{2}\right)-k \rho_{2} \int_{0}^{\zeta_{2}} e^{-k\left(\zeta_{2}-s\right)}\left(I^{\beta-1} \phi\right)(s) d s-\int_{0}^{T}\left(I^{\alpha-1} \psi\right)(s) d s\right. \\
& \left.+k \int_{0}^{T} \int_{0}^{x} e^{-k(x-s)}\left(I^{\alpha-1} \psi\right)(s) d s d x\right],
\end{aligned}
$$

and:

$$
\begin{aligned}
b_{0}=\theta\left[\mu_{2}\left(I^{\alpha-1} \psi\right)\right. & \left(\eta_{2}\right)-k \mu_{2} \int_{0}^{\eta_{2}} e^{-k\left(\eta_{2}-s\right)}\left(I^{\alpha-1} \psi\right)(s) d s \\
& \left.-\int_{0}^{T}\left(I^{\beta-1} \phi\right)(s) d s+k \int_{0}^{T} \int_{0}^{x} e^{-k(x-s)}\left(I^{\beta-1} \phi\right)(s) d s d x\right] \\
& +\tau\left[\rho_{2}\left(I^{\beta-1} \phi\right)\left(\zeta_{2}\right)-k \rho_{2} \int_{0}^{\zeta_{2}} e^{-k\left(\zeta_{2}-s\right)}\left(I^{\beta-1} \phi\right)(s) d s-\int_{0}^{T}\left(I^{\alpha-1} \psi\right)(s) d s\right. \\
& \left.+k \int_{0}^{T} \int_{0}^{x} e^{-k(x-s)}\left(I^{\alpha-1} \psi\right)(s) d s d x\right]
\end{aligned}
$$


In view of the conditions $\int_{0}^{T} x(s) d s=\rho_{1} y\left(\zeta_{1}\right), \int_{0}^{T} y(s) d s=\mu_{1} x\left(\eta_{1}\right)$, we get:

$$
\begin{aligned}
a_{1}=\frac{1}{\omega}\left[\frac{A \mu_{2}}{k}\right. & \left(I^{\alpha-1} \psi\right)\left(\eta_{2}\right)-A \mu_{2} \int_{0}^{\eta_{2}} e^{-k\left(\eta_{2}-s\right)}\left(I^{\alpha-1} \psi\right)(s) d s-\frac{A}{k} \int_{0}^{T}\left(I^{\beta-1} \phi\right)(s) d s \\
& +\left(A-\rho_{1}\right) \int_{0}^{T} \int_{0}^{x} e^{-k(x-s)}\left(I^{\beta-1} \phi\right)(s) d s d x+\frac{B \rho_{2}}{k}\left(I^{\beta-1} \phi\right)\left(\zeta_{2}\right) \\
& -B \rho_{2} \int_{0}^{\zeta_{2}} e^{-k\left(\zeta_{2}-s\right)}\left(I^{\beta-1} \phi\right)(s) d s-\frac{B}{k} \int_{0}^{T}\left(I^{\alpha-1} \psi\right)(s) d s \\
& +(B-T) \int_{0}^{T} \int_{0}^{x} e^{-k(x-s)}\left(I^{\alpha-1} \psi\right)(s) d s d x+T \rho_{1} \int_{0}^{\zeta_{1}} e^{-k\left(\zeta_{1}-s\right)}\left(I^{\beta-1} \phi\right)(s) d s \\
& \left.+\mu_{1} \rho_{1} \int_{0}^{\eta_{1}} e^{-k\left(\eta_{1}-s\right)}\left(I^{\alpha-1} \psi\right)(s) d s\right],
\end{aligned}
$$

and:

$$
\begin{aligned}
b_{1}=\frac{1}{\omega}\left[\frac{C \mu_{2}}{k}\right. & \left(I^{\alpha-1} \psi\right)\left(\eta_{2}\right)-C \mu_{2} \int_{0}^{\eta_{2}} e^{-k\left(\eta_{2}-s\right)}\left(I^{\alpha-1} \psi\right)(s) d s-\frac{C}{k} \int_{0}^{T}\left(I^{\beta-1} \phi\right)(s) d s \\
& +(C-T) \int_{0}^{T} \int_{0}^{x} e^{-k(x-s)}\left(I^{\beta-1} \phi\right)(s) d s d x+\frac{D \rho_{2}}{k}\left(I^{\beta-1} \phi\right)\left(\zeta_{2}\right) \\
& -D \rho_{2} \int_{0}^{\zeta_{2}} e^{-k\left(\zeta_{2}-s\right)}\left(I^{\beta-1} \phi\right)(s) d s-\frac{D}{k} \int_{0}^{T}\left(I^{\alpha-1} \psi\right)(s) d s \\
& +\left(D-\mu_{1}\right) \int_{0}^{T} \int_{0}^{x} e^{-k(x-s)}\left(I^{\alpha-1} \psi\right)(s) d s d x+\mu_{1} \rho_{1} \int_{0}^{\zeta_{1}} e^{-k\left(\zeta_{1}-s\right)}\left(I^{\beta-1} \phi\right)(s) d s \\
& \left.+T \mu_{1} \int_{0}^{\eta_{1}} e^{-k\left(\eta_{1}-s\right)}\left(I^{\alpha-1} \psi\right)(s) d s\right]
\end{aligned}
$$

Substituting the values of $a_{0}, a_{1}, b_{0}, b_{1}$ in (6), (7) we obtain (4) and (5), which completes the proof.

\section{Existence Results}

Let the space $Q=\{x(t) \mid x(t) \in C[0, T]\}$, endowed with the norm $\|x\|=\max \{|x(t)|$, $t \in[0, T]\}$. It is clear that $(Q,\|\|$.$) is a Banach space. Moreover, let S=\{y(t) \mid y(t) \in C[0, T]\}$, endowed with the norm $\|y\|=\max \{|y(t)|, t \in[0, T]\}$. The product space $(Q \times S,\|(x, y)\|)$ is also a Banach space with the norm $\|(x, y)\|=\|x\|+\|y\|$.

In view of Lemma 1 we define the operator $Z: Q \times S \rightarrow Q \times S$ by:

$$
Z(x, y)(t)=\left(Z_{1}(x, y)(t), Z_{2}(x, y)(t)\right)
$$


where:

$$
\begin{aligned}
Z_{1}(x, y)(t)= & \Delta e^{-k t}\left[\mu_{2}\left(I^{\alpha-1} f\right)\left(\eta_{2}\right)-k \mu_{2} \int_{0}^{\eta_{2}} e^{-k\left(\eta_{2}-s\right)}\left(I^{\alpha-1} f\right)(s) d s\right. \\
& \left.-\int_{0}^{T}\left(I^{\beta-1} g\right)(s) d s+k \int_{0}^{T} \int_{0}^{x} e^{-k(x-s)}\left(I^{\beta-1} g\right)(s) d s d x\right] \\
& +\lambda e^{-k t}\left[\rho_{2}\left(I^{\beta-1} g\right)\left(\zeta_{2}\right)-k \rho_{2} \int_{0}^{\zeta_{2}} e^{-k\left(\zeta_{2}-s\right)}\left(I^{\beta-1} g\right)(s) d s-\int_{0}^{T}\left(I^{\alpha-1} f\right)(s) d s\right. \\
& \left.+k \int_{0}^{T} \int_{0}^{x} e^{-k(x-s)}\left(I^{\alpha-1} f\right)(s) d s d x\right] \\
& +\frac{1}{\omega}\left[\frac{A \mu_{2}}{k}\left(I^{\alpha-1} f\right)\left(\eta_{2}\right)-A \mu_{2} \int_{0}^{\eta_{2}} e^{-k\left(\eta_{2}-s\right)}\left(I^{\alpha-1} f\right)(s) d s-\frac{A}{k} \int_{0}^{T}\left(I^{\beta-1} g\right)(s) d s\right. \\
& +\left(A-\rho_{1}\right) \int_{0}^{T} \int_{0}^{x} e^{-k(x-s)}\left(I^{\beta-1} g\right)(s) d s d x+\frac{B \rho_{2}}{k}\left(I^{\beta-1} g\right)\left(\zeta_{2}\right) \\
& -B \rho_{2} \int_{0}^{\zeta_{2}} e^{-k\left(\zeta_{2}-s\right)}\left(I^{\beta-1} g\right)(s) d s-\frac{B}{k} \int_{0}^{T}\left(I^{\alpha-1} f\right)(s) d s \\
& +(B-T) \int_{0}^{T} \int_{0}^{x} e^{-k(x-s)}\left(I^{\alpha-1} f\right)(s) d s d x+T \rho_{1} \int_{0}^{\zeta_{1}} e^{-k\left(\zeta_{1}-s\right)}\left(I^{\beta-1} g\right)(s) d s \\
& \left.+\mu_{1} \rho_{1} \int_{0}^{\eta_{1}} e^{-k\left(\eta_{1}-s\right)}\left(I^{\alpha-1} f\right)(s) d s\right]+\int_{0}^{t} e^{-k(t-s)}\left(I^{\alpha-1} f\right)(s) d s,
\end{aligned}
$$

and:

$$
\begin{aligned}
Z_{2}(x, y)(t)= & \theta e^{-k t}\left[\mu_{2}\left(I^{\alpha-1} f\right)\left(\eta_{2}\right)-k \mu_{2} \int_{0}^{\eta_{2}} e^{-k\left(\eta_{2}-s\right)}\left(I^{\alpha-1} f\right)(s) d s\right. \\
& \left.-\int_{0}^{T}\left(I^{\beta-1} g\right)(s) d s+k \int_{0}^{T} \int_{0}^{x} e^{-k(x-s)}\left(I^{\beta-1} g\right)(s) d s d x\right] \\
& +\tau e^{-k t}\left[\rho_{2}\left(I^{\beta-1} g\right)\left(\zeta_{2}\right)-k \rho_{2} \int_{0}^{\zeta_{2}} e^{-k\left(\zeta_{2}-s\right)}\left(I^{\beta-1} g\right)(s) d s-\int_{0}^{T}\left(I^{\alpha-1} f\right)(s) d s\right. \\
& \left.+k \int_{0}^{T} \int_{0}^{x} e^{-k(x-s)}\left(I^{\alpha-1} f\right)(s) d s d x\right] \\
& +\frac{1}{\omega}\left[\frac{C \mu_{2}}{k}\left(I^{\alpha-1} f\right)\left(\eta_{2}\right)-C \mu_{2} \int_{0}^{\eta_{2}} e^{-k\left(\eta_{2}-s\right)}\left(I^{\alpha-1} f\right)(s) d s-\frac{C}{k} \int_{0}^{T}\left(I^{\beta-1} g\right)(s) d s\right. \\
& +(C-T) \int_{0}^{T} \int_{0}^{x} e^{-k(x-s)}\left(I^{\beta-1} g\right)(s) d s d x+\frac{D \rho_{2}}{k}\left(I^{\beta-1} g\right)\left(\zeta_{2}\right) \\
& -D \rho_{2} \int_{0}^{\zeta_{2}} e^{-k\left(\zeta_{2}-s\right)}\left(I^{\beta-1} g\right)(s) d s-\frac{D}{k} \int_{0}^{T}\left(I^{\alpha-1} f\right)(s) d s \\
& +\left(D-\mu_{1}\right) \int_{0}^{T} \int_{0}^{x} e^{-k(x-s)}\left(I^{\alpha-1} f\right)(s) d s d x+\mu_{1} \rho_{1} \int_{0}^{\zeta_{1}} e^{-k\left(\zeta_{1}-s\right)}\left(I^{\beta-1} g\right)(s) d s \\
& \left.+T \mu_{1} \int_{0}^{\eta_{1}} e^{-k\left(\eta_{1}-s\right)}\left(I^{\alpha-1} f\right)(s) d s\right]+\int_{0}^{t} e^{-k(t-s)}\left(I^{\beta-1} g\right)(s) d s .
\end{aligned}
$$

Theorem 1. Assume $f, g: C\left([0, T] \times \mathbb{R}^{2} \rightarrow \mathbb{R}\right.$ are jointly continuous functions, and there exists constants $h_{1}, h_{2} \in \mathbb{R}$, such that $\forall x_{1}, x_{2}, y_{1}, y_{2} \in \mathbb{R}, \forall t \in[0, T]$ we have:

$$
\begin{aligned}
& \left|f\left(t, x_{1}, x_{2}\right)-f\left(t, y_{1}, y_{2}\right)\right| \leq h_{1}\left(\left|x_{2}-x_{1}\right|+\left|y_{2}-y_{1}\right|\right), \\
& \left|g\left(t, x_{1}, x_{2}\right)-f\left(t, y_{1}, y_{2}\right)\right| \leq h_{2}\left(\left|x_{2}-x_{1}\right|+\left|y_{2}-y_{1}\right|\right) .
\end{aligned}
$$


If:

$$
h_{1}\left(M_{1}+M_{3}\right)+h_{2}\left(M_{2}+M_{4}\right)<1,
$$

then the boundary value problems (1) and (2) have a unique solution on $[0, T]$, where:

$$
\begin{gathered}
M_{1}=\left[\frac{(\alpha+1)\left|\Delta \mu_{2}\right| e^{-k T} \eta_{2}{ }^{\alpha}\left(\alpha \eta_{2}{ }^{-1}+k\right)+|\lambda| e^{-k T} T^{\alpha}(\alpha+1+k T)}{\Gamma(\alpha+2)}\right]+\frac{T^{\alpha}}{\Gamma(\alpha+1)}+ \\
{\left[\frac{\left|A \mu_{2}\right| \eta_{2}{ }^{\alpha}(\alpha+1)\left(\alpha \eta_{2}{ }^{-1}+k\right)+T^{\alpha}(|B|(\alpha+1)+k T|B-T|)+k(\alpha+1)\left|\mu_{1} \rho_{1}\right| \eta_{1}{ }^{\alpha}}{k \Gamma(\alpha+2)\left|T^{2}-\mu_{1} \rho_{1}\right|}\right],} \\
M_{2}=\left[\frac{(\beta+1) T^{\beta}\left(|\Delta| e^{-k T}+k T\right)+\left|\lambda \rho_{2}\right| e^{-k T} \zeta_{2}{ }^{\beta}(\beta+1)\left(\beta \zeta_{2}{ }^{-1}+k\right)}{\Gamma(\beta+2)}\right]+ \\
{\left[\frac{T^{\beta-1}\left(|A|\left(\beta^{2}+\beta\right)+k\left|A-\rho_{1}\right| T^{2}\right)+(\beta+1)\left|B \rho_{2}\right| \zeta_{2} \beta\left(\beta \zeta_{2}{ }^{-1}+k\right)+k\left|T \rho_{1}\right| \zeta_{1}^{\beta}}{k \Gamma(\beta+2)\left|T^{2}-\mu_{1} \rho_{1}\right|}\right],} \\
{\left[\frac{\left|C \mu_{2}\right| \eta_{2}{ }^{\alpha}(\alpha+1)\left(\alpha \eta_{2}{ }^{-1}+k\right)+T^{\alpha}\left(|D|(\alpha+1)+k T\left|D-\mu_{1}\right|\right)+k(\alpha+1)\left|\mu_{1} T\right| \eta_{1}{ }^{\alpha}}{k \Gamma(\alpha+2)\left|T^{2}-\mu_{1} \rho_{1}\right|}\right],} \\
M_{4}=\left[\frac{(\beta+1) T^{\beta}\left(|\theta| e^{-k T}+k T\right)+\left|\tau \rho_{2}\right| e^{-k T} \zeta_{2}{ }^{\beta}(\beta+1)\left(\beta \zeta_{2}{ }^{-1}+k\right)}{\Gamma(\beta+2)}\right]+\frac{T^{\beta}}{\Gamma(\beta+1)}+ \\
{\left[\frac{T^{\beta-1}\left(|C|\left(\beta^{2}+\beta\right)+k|C-T| T^{2}\right)+(\beta+1)\left|D \rho_{2}\right| \zeta_{2}{ }^{\beta}\left(\beta \zeta_{2}{ }^{-1}+k\right)+k\left|\mu_{1} \rho_{1}\right| \zeta_{1}^{\beta}}{k \Gamma(\beta+2)\left|T^{2}-\mu_{1} \rho_{1}\right|}\right] .}
\end{gathered}
$$

Proof. Define $\sup _{0 \leq t \leq T} f(t, 0,0)=f_{0}<\infty, \quad \sup _{0 \leq t \leq T} g(t, 0,0)=g_{0}<\infty$ and $\Omega_{\varepsilon}=\{(x, y) \in Q \times S:(x, y) \leq \varepsilon\}$ and $\varepsilon>0$ such that:

$$
\varepsilon \geq \frac{\left(M_{1}+M_{3}\right) f_{0}+\left(M_{2}+M_{4}\right) g_{0}}{1-\left[h_{1}\left(M_{1}+M_{3}\right)+h_{2}\left(M_{2}+M_{4}\right)\right]} .
$$

Firstly, show that $Z \Omega_{\varepsilon} \subseteq \Omega_{\mathcal{\varepsilon}}$.

By our assumption, for $(x, y) \in \Omega_{\varepsilon}, t \in[0, T]$, we have:

$$
\begin{gathered}
|f(t, x(t), y(t))| \leq|f(t, x(t), y(t))-f(t, 0,0)|+|f(t, 0,0)|, \\
\leq h_{1}(|x(t)|+|y(t)|)+f_{0} \leq h_{1}(x+y)+f_{0}, \\
\leq h_{1} \varepsilon+f_{0}
\end{gathered}
$$

and:

$$
\begin{gathered}
|g(t, x(t), y(t))| \leq h_{2}(|x(t)|+|y(t)|)+g_{0} \leq h_{2}(x+y)+g_{0}, \\
\leq h_{2} \varepsilon+g_{0},
\end{gathered}
$$




\section{which leads to:}

$$
\begin{aligned}
\left|Z_{1}(x, y)(t)\right| \leq & |\Delta| e^{-k T}\left[\left|\mu_{2}\right|\left(I^{\alpha-1}|f|\right)\left(\eta_{2}\right)+k\left|\mu_{2}\right| \int_{0}^{\eta_{2}} e^{-k\left(\eta_{2}-s\right)}\left(I^{\alpha-1}|f|\right)(s) d s\right. \\
& \left.+\int_{0}^{T}\left(I^{\beta-1}|g|\right)(s) d s+k \int_{0}^{T} \int_{0}^{x} e^{-k(x-s)}\left(I^{\beta-1}|g|\right)(s) d s d x\right] \\
& +|\lambda| e^{-k T}\left[\left|\rho_{2}\right|\left(I^{\beta-1}|g|\right)\left(\zeta_{2}\right)+k\left|\rho_{2}\right| \int_{0}^{\zeta_{2}} e^{-k\left(\zeta_{2}-s\right)}\left(I^{\beta-1}|g|\right)(s) d s+\int_{0}^{T}\left(I^{\alpha-1}|f|\right)(s) d s\right. \\
& \left.+k \int_{0}^{T} \int_{0}^{x} e^{-k(x-s)}\left(I^{\alpha-1}|f|\right)(s) d s d x\right] \\
& +\frac{1}{|\omega|}\left[\frac{\left|A \mu_{2}\right|}{k}\left(I^{\alpha-1}|f|\right)\left(\eta_{2}\right)+\left|A \mu_{2}\right| \int_{0}^{\eta_{2}} e^{-k\left(\eta_{2}-s\right)}\left(I^{\alpha-1}|f|\right)(s) d s+\frac{|A|}{k} \int_{0}^{T}\left(I^{\beta-1}|g|\right)(s) d s\right. \\
& +\left|A-\rho_{1}\right| \int_{0}^{T} \int_{0}^{x} e^{-k(x-s)}\left(I^{\beta-1}|g|\right)(s) d s d x+\frac{\left|B \rho_{2}\right|}{k}\left(I^{\beta-1}|g|\right)\left(\zeta_{2}\right) \\
& +\left|B \rho_{2}\right| \int_{0}^{\zeta_{2}} e^{-k\left(\zeta_{2}-s\right)}\left(I^{\beta-1}|g|\right)(s) d s+\frac{|B|}{k} \int_{0}^{T}\left(I^{\alpha-1}|f|\right)(s) d s \\
& +|B-T| \int_{0}^{T} \int_{0}^{x} e^{-k(x-s)}\left(I^{\alpha-1}|f|\right)(s) d s d x \\
& \left.+T\left|\rho_{1}\right| \int_{0}^{\zeta 1} e^{-k\left(\zeta_{1}-s\right)}\left(I^{\beta-1}|g|\right)(s) d s+\left|\mu_{1} \rho_{1}\right| \int_{0}^{\eta_{2}} e^{-k\left(\eta_{2}-s\right)}\left(I^{\alpha-1}|f|\right)(s) d s\right] \\
& +0 \leq t \leq T \int_{0}^{t} e^{-k(t-s)}\left(I^{\alpha-1}|f|\right)(s) d s .
\end{aligned}
$$

Using (10) and (11) to get:

$$
\begin{aligned}
\left|Z_{1}(x, y)(t)\right| \leq & {\left[|\Delta| e^{-k T} \mu_{2}\left(\left(I^{\alpha-1} 1\right)\left(\eta_{2}\right)+k \int_{0}^{\eta_{2}} e^{-k\left(\eta_{2}-s\right)}\left(I^{\alpha-1} 1\right)(s) d s\right)\right.} \\
& +|\lambda| e^{-k T}\left(\int_{0}^{T}\left(I^{\alpha-1} 1\right)(s) d s+k \int_{0}^{T} \int_{0}^{x} e^{-k(x-s)}\left(I^{\alpha-1} 1\right)(s) d s d x\right) \\
& +\frac{1}{|\omega|}\left(\frac{\left|A \mu_{2}\right|}{k}\left(I^{\alpha-1} 1\right)\left(\eta_{2}\right)+\left|A \mu_{2}\right| \int_{0}^{\eta_{2}} e^{-k\left(\eta_{2}-s\right)}\left(I^{\alpha-1} 1\right)(s) d s+\frac{|B|}{k} \int_{0}^{T}\left(I^{\alpha-1} 1\right)(s) d s\right. \\
& \left.+|B-T| \int_{0}^{T} \int_{0}^{x} e^{-k(x-s)}\left(I^{\alpha-1} 1\right)(s) d s d x+\left|\mu_{1} \rho_{1}\right| \int_{0}^{\eta_{1}} e^{-k\left(\eta_{1}-s\right)}\left(I^{\alpha-1} 1\right)(s) d s\right) \\
& \left.+\int_{0}^{T} e^{-k(T-s)}\left(I^{\alpha-1} 1\right)(s) d s\right]|| f|| \\
& +\left[|\Delta| e^{-k T}\left(\int_{0}^{T}\left(I^{\beta-1} 1\right)(s) d s+k \int_{0}^{T} \int_{0}^{x} e^{-k(x-s)}\left(I^{\beta-1} 1\right)(s) d s d x\right)\right. \\
& +|\lambda| e^{-k T}\left(\left|\rho_{2}\right|\left(I^{\beta-1} 1\right)\left(\zeta_{2}\right)+k\left|\rho_{2}\right| \int_{0}^{\zeta_{2}} e^{-k\left(\zeta_{2}-s\right)}\left(I^{\beta-1} 1\right)(s) d s\right) \\
& +\frac{1}{|\omega|}\left(\frac{|A|}{k} \int_{0}^{T}\left(I^{\beta-1} 1\right)(s) d s+\left|A-\rho_{1}\right| \int_{0}^{T} \int_{0}^{x} e^{-k(x-s)}\left(I^{\beta-1} 1\right)(s) d s d x+\frac{\left|B \rho_{2}\right|}{k}\left(I^{\beta-1} 1\right)\left(\zeta_{2}\right)\right. \\
& \left.\left.+\left|B \rho_{2}\right| \int_{0}^{\zeta_{2}} e^{-k\left(\zeta_{2}-s\right)}\left(I^{\beta-1} 1\right)(s) d s\right)\right]|| g||
\end{aligned}
$$




$$
\begin{aligned}
\leq & {\left[\frac{(\alpha+1)\left|\Delta \mu_{2}\right| e^{-k T} \eta_{2}{ }^{\alpha}\left(\alpha \eta_{2}{ }^{-1}+k\right)+|\lambda| e^{-k T} T^{\alpha}(\alpha+1+k T)}{\Gamma(\alpha+2)}+\frac{T^{\alpha}}{\Gamma(\alpha+1)}\right.} \\
& \left.+\frac{\left|A \mu_{2}\right| \eta_{2}{ }^{\alpha}(\alpha+1)\left(\alpha \eta_{2}{ }^{-1}+k\right)+T^{\alpha}(|B|(\alpha+1)+k T|B-T|)+k(\alpha+1)\left|\mu_{1} \rho_{1}\right| \eta_{1}^{\alpha}}{k \Gamma(\alpha+2)|\omega|}\right]\|f\| \\
& +\left[\frac{(\beta+1) T^{\beta}\left(|\Delta| e^{-k T}+k T\right)+\left|\lambda \rho_{2}\right| e^{-k T} \zeta_{2}{ }^{\beta}(\beta+1)\left(\beta \zeta_{2}{ }^{-1}+k\right)}{\Gamma(\beta+2)}\right. \\
& \left.+\frac{T^{\beta-1}\left(|A|\left(\beta^{2}+\beta\right)+k\left|A-\rho_{1}\right| T^{2}\right)+(\beta+1)\left|B \rho_{2}\right| \zeta_{2}^{\beta}\left(\beta \zeta_{2}{ }^{-1}+k\right)+k\left|T \rho_{1}\right| \zeta_{1}^{\beta}}{k \Gamma(\alpha+2)|\omega|}\right]\|g\|
\end{aligned}
$$

Hence, by (8) we have:

$$
\left\|Z_{1}(x, y)\right\| \leq\left(h_{1} M_{1}+h_{2} M_{2}\right) \varepsilon+\left(M_{1} f_{0}+M_{2} g_{0}\right) \leq \frac{\varepsilon}{2} .
$$

In similar way, we get:

$$
\left\|Z_{2}(x, y)\right\| \leq\left(h_{1} M_{3}+h_{2} M_{4}\right) r+\left(M_{3} f_{0}+M_{4} g_{0}\right) \leq \frac{\varepsilon}{2} .
$$

From (12) and (13), we obtain:

$$
\|Z(x, y)\| \leq \varepsilon .
$$

Now, show that $Z$ is a contraction.

Let $\left(x_{1}, y_{1}\right),\left(x_{2}, y_{2}\right) \in Q \times S, \forall t \in[0, T]$, then we get:

$$
\begin{gathered}
\left\|Z_{1}\left(x_{1}, y_{1}\right)-Z_{1}\left(x_{2}, y_{2}\right)\right\| \leq h_{1} M_{1}\left(\left\|x_{1}-x_{2}\right\|+\left\|y_{1}-y_{2}\right\|\right)+h_{2} M_{2}\left(\left\|x_{1}-x_{2}\right\|+\left\|y_{1}-y_{2}\right\|\right), \\
\left\|Z_{2}\left(x_{1}, y_{1}\right)-Z_{2}\left(x_{2}, y_{2}\right)\right\| \leq h_{1} M_{3}\left(\left\|x_{1}-x_{2}\right\|+\left\|y_{1}-y_{2}\right\|\right)+h_{2} M_{4}\left(\left\|x_{1}-x_{2}\right\|+\left\|y_{1}-y_{2}\right\|\right) .
\end{gathered}
$$

From (14) and (15), we deduced that:

$$
\left\|Z\left(x_{1}, y_{1}\right)-Z\left(x_{2}, y_{2}\right)\right\| \leq\left(h_{1}\left(M_{1}+M_{3}\right)+h_{2}\left(M_{2}+M_{4}\right)\right)\left(\left\|x_{1}-x_{2}\right\|+\left\|y_{1}-y_{2}\right\|\right) .
$$

Since $h_{1}\left(M_{1}+M_{3}\right)+h_{2}\left(M_{2}+M_{4}\right)<1$, therefore, $Z$ is a contraction operator. Thus, by Banach's fixed point theorem, the operator $Z$ has a unique fixed point on $[0, T]$, which is the unique solution of the problem (1) and (2), and completes the proof.

The second result is based on the Leray-Schauder alternative.

Lemma 2 (Leray-Schauder alternative [18], p. 4). Let $F: E \rightarrow E$ be a completely continuous operator (i.e., a map restricted to any bounded set in $E$ is compact). Let $E(F)=$ $\{x \in E: x=\lambda F(x)$ for some $0<\lambda<1\}$. Then, either the set $E(F)$ is unbounded, or $F$ has at least one fixed point.

Theorem 2. Assume $f, g: C\left([0, T] \times \mathbb{R}^{2} \rightarrow \mathbb{R}\right.$ are continuous functions and there exists positive real constants $\theta_{i}, \vartheta_{i}(i=0,1,2)$ such that $\forall x_{i} \in \mathbb{R},(i=1,2)$ we have:

$$
\begin{aligned}
& \left|f\left(t, x_{1}, x_{2}\right)\right| \leq \theta_{0}+\theta_{1}\left|x_{1}\right|+\theta_{2}\left|x_{2}\right|, \\
& \left|g\left(t, x_{1}, x_{2}\right)\right| \leq \vartheta_{0}+\vartheta_{1}\left|x_{1}\right|+\vartheta_{2}\left|x_{2}\right| .
\end{aligned}
$$

If:

$$
\left(M_{1}+M_{3}\right) \theta_{1}+\left(M_{2}+M_{4}\right) \vartheta_{1}<1
$$

and:

$$
\left(M_{1}+M_{3}\right) \theta_{2}+\left(M_{2}+M_{4}\right) \vartheta_{2}<1
$$


Then the problems (1) and (2) have at least one solution.

Proof. The proof will be divided into several steps [24-26].

Step 1. Show that $Z$ is completely continuous. The continuity of the operator holds true because of the continuity of the function $f, g$.

Let $\mathrm{R}$ be a bounded set in $\Omega_{\varepsilon}=\{(\mathrm{x}, \mathrm{y}) \in \mathrm{Q} \times \mathrm{S}:\|(\mathrm{x}, \mathrm{y})\| \leq \varepsilon\}$. Then, there exists positive constants $\mathrm{n}_{1}, \mathrm{n}_{2}$ such that:

$$
|f(t, x(t), y(t))| \leq n_{1},|g(t, x(t), y(t))| \leq n_{2}, \forall t \in[0, T],
$$

then, for any $(x, y) \in R$ we have $\left|Z_{1}(x, y)(t)\right| \leq M_{1} n_{1}+M_{2} n_{2}$, which implies that:

$$
\left\|Z_{1}(x, y)\right\| \leq M_{1} n_{1}+M_{2} n_{2} .
$$

Similarly, we get $\left\|Z_{2}(x, y)\right\| \leq M_{3} n_{1}+M_{4} n_{2}$.

Thus, it follows from the above inequalities that the operator $Z$ is uniformly bounded, since:

$$
\|Z(x, y)\| \leq\left(M_{1}+M_{3}\right) n_{1}+\left(M_{2}+M_{4}\right) n_{2} .
$$

Next, we show that the operator is equicontinuous.

Let $t_{1}, t_{2} \in[0, T]$ with $t_{1}<t_{2}$. Then we have:

$$
\begin{aligned}
\mid Z_{1}(x, y)\left(\mathrm{t}_{2}\right)- & Z_{1}(x, y)\left(\mathrm{t}_{1}\right) \mid \\
\leq & |\Delta| e^{-k\left(\mathrm{t}_{2}-\mathrm{t}_{1}\right)}\left[\left|\mu_{2}\right|\left(I^{\alpha-1}|f|\right)\left(\eta_{2}\right)+k\left|\mu_{2}\right| \int_{0}^{\eta_{2}} e^{-k\left(\eta_{2}-s\right)}\left(I^{\alpha-1}|f|\right)(s) d s\right. \\
& \left.+\int_{0}^{T}\left(I^{\beta-1}|g|\right)(s) d s+k \int_{0}^{T} \int_{0}^{x} e^{-k(x-s)}\left(I^{\beta-1}|g|\right)(s) d s d x\right] \\
& +|\lambda| e^{-k\left(\mathrm{t}_{2}-\mathrm{t}_{1}\right)}\left[\left|\rho_{2}\right|\left(I^{\beta-1}|g|\right)\left(\zeta_{2}\right)+k\left|\rho_{2}\right| \int_{0}^{\zeta_{2}} e^{-k\left(\zeta_{2}-s\right)}\left(I^{\beta-1}|g|\right)(s) d s+\int_{0}^{T}\left(I^{\alpha-1}|f|\right)(s) d s\right. \\
+ & \left.k \int_{0}^{T} \int_{0}^{x} e^{-k(x-s)}\left(I^{\alpha-1}|f|\right)(s) d s d x\right]+\int_{0}^{\mathrm{t}_{2}} e^{-k\left(\mathrm{t}_{2}-s\right)}\left(I^{\alpha-1}|f|\right)(s) d s \\
+ & \int_{0}^{\mathrm{t}_{1}} e^{-k\left(\mathrm{t}_{1}-s\right)}\left(I^{\alpha-1}|f|\right)(s) d s . \\
\leq|\Delta| e^{-k\left(\mathrm{t}_{2}-\mathrm{t}_{1}\right)}[ & {\left[\mu_{2} n_{1}\left|\left(I^{\alpha-1} 1\right)\left(\eta_{2}\right)+k\right| \mu_{2} n_{1} \mid \int_{0}^{\eta_{2}} e^{-k\left(\eta_{2}-s\right)}\left(I^{\alpha-1} 1\right)(s) d s\right.} \\
& \left.+n_{2} \int_{0}^{T}\left(I^{\beta-1} 1\right)(s) d s+k n_{2} \int_{0}^{T} \int_{0}^{x} e^{-k(x-s)}\left(I^{\beta-1} 1\right)(s) d s d x\right] \\
& +|\lambda| e^{-k\left(\mathrm{t}_{2}-\mathrm{t}_{1}\right)}\left[\left|\rho_{2} n_{2}\right|\left(I^{\beta-1} 1\right)\left(\zeta_{2}\right)+k\left|\rho_{2} n_{2}\right| \int_{0}^{\zeta_{2}} e^{-k\left(\zeta_{2}-s\right)}\left(I^{\beta-1} 1\right)(s) d s\right. \\
& \left.+n_{1} \int_{0}^{T}\left(I^{\alpha-1} 1\right)(s) d s+k n_{1} \int_{0}^{T} \int_{0}^{x} e^{-k(x-s)}\left(I^{\alpha-1} 1\right)(s) d s d x\right] \\
& +n_{1}\left[\left(\int_{0}^{\mathrm{t}_{1}} e^{-k\left(\mathrm{t}_{2}-s\right)}-e^{-k\left(\mathrm{t}_{1}-s\right)}\right)\left(I^{\alpha-1} 1\right)(s) d s+\int_{\mathrm{t}_{1}}^{\mathrm{t}_{2}} e^{-k\left(\mathrm{t}_{2}-s\right)}\left(I^{\alpha-1} 1\right)(s) d s\right]
\end{aligned}
$$

Hence, we have $\left\|Z_{1}(x, y)\left(t_{2}\right)-Z_{1}(x, y)\left(t_{1}\right)\right\| \rightarrow 0$ independent of $x$ and $y$ as $t_{2} \rightarrow t_{1}$. Similarly, $\left\|Z_{2}(x, y)\left(t_{2}\right)-Z_{2}(x, y)\left(t_{1}\right)\right\| \rightarrow 0$ independent of $x$ and $y$ as $t_{2} \rightarrow t_{1}$.

Therefore, the operator $Z(x, y)$ is equicontinuous, and thus the operator $Z(x, y)$ is completely continuous.

Step 2. Boundedness of operator.

Finally, show that $r=\{(x, y) \in Q \times S:(x, y)=M Z(x, y), N \in[0,1]\}$ is bounded.

Let:

$$
x(t)=M Z_{1}(x, y)(t), y(t)=M Z_{2}(x, y)(t) .
$$


Then:

$$
|x(t)| \leq M_{1}\left(\theta_{0}+\theta_{1}|x|+\theta_{2}|y|\right)+N_{2}\left(\vartheta_{0}+\vartheta_{1}|x|+\vartheta_{2}|y|\right),
$$

and:

$$
|y(t)| \leq M_{3}\left(\theta_{0}+\theta_{1}|x|+\theta_{2}|y|\right)+N_{4}\left(\vartheta_{0}+\vartheta_{1}|x|+\vartheta_{2}|y|\right) .
$$

So, we get:

$$
\|x\| \leq M_{1}\left(\theta_{0}+\theta_{1}|x|+\theta_{2}|y|\right)+M_{2}\left(\vartheta_{0}+\vartheta_{1}|x|+\vartheta_{2}|y|\right),
$$

and:

$$
\|y\| \leq M_{3}\left(\theta_{0}+\theta_{1}|x|+\theta_{2}|y|\right)+M_{4}\left(\vartheta_{0}+\vartheta_{1}|x|+\vartheta_{2}|y|\right) .
$$

From (16) and (17), we obtain:

$$
\begin{gathered}
\|x\|+\|y\| \leq\left(M_{1}+M_{3}\right) \theta_{0}+\left(M_{2}+M_{4}\right) \vartheta_{0}+\left(\left(M_{1}+M_{3}\right) \theta_{1}+\left(M_{2}+M_{4}\right) \vartheta_{1}\right)\|x\| \\
+\left(\left(M_{1}+M_{3}\right) \theta_{2}+\left(M_{2}+M_{4}\right) \vartheta_{2}\right)\|y\| .
\end{gathered}
$$

Therefore:

$$
\|(x, y)\| \leq \frac{\left(M_{1}+M_{3}\right) \theta_{0}+\left(M_{2}+M_{4}\right) \vartheta_{0}}{M_{0}},
$$

where $M_{0}=\min \left\{1-\left(M_{1}+M_{3}\right) \theta_{1}-\left(M_{2}+M_{4}\right) \vartheta_{1}, 1-\left(M_{1}+M_{3}\right) \theta_{2}-\left(M_{2}+M_{4}\right) \vartheta_{2}\right\}$ that is $r$ bounded. By the Leray-Schauder theorem, the existence of a solution to the boundary value problem holds true on $[0, T]$.

\section{Ulam-Hyers Stability}

The Ulam-Hyers stability for our suggested system (1) will be investigated by considering the below inequality:

$$
\left\{\begin{array}{l}
{ }^{c} D^{\alpha-1}(D+k) x(t)-f(t, x(t), y(t)) \leq \varepsilon_{1}, t \in[0, T] \\
{ }^{c} D^{\beta-1}(D+k) y(t)-g(t, x(t), y(t)) \leq \varepsilon_{2}, t \in[0, T]
\end{array}\right.
$$

where $\varepsilon_{1}, \varepsilon_{2}$ are given two positive real numbers.

Define the following nonlinear operators $N_{1}, N_{2} \in C([0, T], \mathbb{R}) \times C([0, T], \mathbb{R}) \rightarrow$ $C([0, T], \mathbb{R})$ :

$$
\begin{aligned}
& { }^{c} D^{\alpha-1}(D+k) x(t)-f(t, x(t), y(t))=N_{1}(t), t \in[0, T], \\
& { }^{c} D^{\beta-1}(D+k) y(t)-g(t, x(t), y(t))=N_{2}(t), t \in[0, T] .
\end{aligned}
$$

For some $\varepsilon_{1}, \varepsilon_{2}>0$, we consider the following inequality:

$$
\left|N_{1}(t)\right| \leq \varepsilon,\left|N_{2}(t)\right| \leq \varepsilon_{2}, t \in[0, T] .
$$

Definition $4[27,28]$. The boundary value problem (1) is Ulam-Hyers stable if there exists $M_{i}, i=1,2,3,4$ such that for the given $\varepsilon_{1}, \varepsilon_{2}>0$ and for each solution $(x, y) \in C\left([0, T] \times \mathbb{R}^{2}, \mathbb{R}\right)$ of inequality (18), there exists a solution $\left(x^{*}, y^{*}\right) \in C\left([0, T] \times \mathbb{R}^{2}, \mathbb{R}\right)$ of problem (1) with:

$$
\left\{\begin{array}{l}
\left|x(t)-x^{*}(t)\right| \leq M_{1} \varepsilon_{1}+M_{2} \varepsilon_{2}, t \in[0, T] \\
\left|y(t)-y^{*}(t)\right| \leq M_{3} \varepsilon_{1}+M_{4} \varepsilon_{2}, t \in[0, T] .
\end{array}\right.
$$

Theorem 3. If the assumptions of Theorem 1 hold, then the BVB (1), (2) is Ulam-Hyers stable. 
Proof. Let $(x, y) \in C([0, T], \mathbb{R}) \times C([0, T], \mathbb{R})$ be the solution of the problem (1) and (2), satisfying (8) and (9), and let $\left(x^{*}, y^{*}\right)$ be any solution satisfying:

$$
\left\{\begin{array}{l}
{ }^{c} D^{\alpha-1}(D+k) x^{*}(t)=f\left(t, x^{*}(t), y^{*}(t)\right)+N_{1}(t), t \in[0, T], \\
{ }^{c} D^{\beta-1}(D+k) y^{*}(t)=g\left(t, x^{*}(t), y^{*}(t)\right)+N_{2}(t), t \in[0, T] .
\end{array}\right.
$$

It follows that:

$$
\begin{aligned}
\mid x(t)-\Delta e^{-k t} & {\left[\mu_{2}\left(I^{\alpha-1} f\right)\left(\eta_{2}\right)-k \mu_{2} \int_{0}^{\eta_{2}} e^{-k\left(\eta_{2}-s\right)}\left(I^{\alpha-1} f\right)(s) d s\right.} \\
& \left.-\int_{0}^{T}\left(I^{\beta-1} g\right)(s) d s+k \int_{0}^{T} \int_{0}^{x} e^{-k(x-s)}\left(I^{\beta-1} g\right)(s) d s d x\right] \\
& -\lambda e^{-k t}\left[\rho_{2}\left(I^{\beta-1} g\right)\left(\zeta_{2}\right)-k \rho_{2} \int_{0}^{\zeta_{2}} e^{-k\left(\zeta_{2}-s\right)}\left(I^{\beta-1} g\right)(s) d s-\int_{0}^{T}\left(I^{\alpha-1} f\right)(s) d s\right. \\
& \left.+k \int_{0}^{T} \int_{0}^{x} e^{-k(x-s)}\left(I^{\alpha-1} f\right)(s) d s d x\right] \\
& -\frac{1}{T^{2}-\mu_{1} \rho_{1}}\left[\frac{A \mu_{2}}{k}\left(I^{\alpha-1} f\right)\left(\eta_{2}\right)-A \mu_{2} \int_{0}^{\eta_{2}} e^{-k\left(\eta_{2}-s\right)}\left(I^{\alpha-1} f\right)(s) d s-\frac{A}{k} \int_{0}^{T}\left(I^{\beta-1} g\right)(s) d s\right. \\
& +\left(A-\rho_{1}\right) \int_{0}^{T} \int_{0}^{x} e^{-k(x-s)}\left(I^{\beta-1} g\right)(s) d s d x+\frac{B \rho_{2}}{k}\left(I^{\beta-1} g\right)\left(\zeta_{2}\right) \\
& -B \rho_{2} \int_{0}^{\zeta_{2}} e^{-k\left(\zeta_{2}-s\right)}\left(I^{\beta-1} g\right)(s) d s-\frac{B}{k} \int_{0}^{T}\left(I^{\alpha-1} f\right)(s) d s \\
& +(B-T) \int_{0}^{T} \int_{0}^{x} e^{-k(x-s)}\left(I^{\alpha-1} f\right)(s) d s d x+T \rho_{1} \int_{0}^{\zeta_{1}} e^{-k\left(\zeta_{1}-s\right)}\left(I^{\beta-1} g\right)(s) d s \\
& \left.+\mu_{1} \rho_{1} \int_{0}^{\eta_{1}} e^{-k\left(\eta_{1}-s\right)}\left(I^{\alpha-1} f\right)(s) d s\right]-\int_{0}^{t} e^{-k(t-s)}\left(I^{\alpha-1} f\right)(s) d s \mid
\end{aligned}
$$$$
\leq|\Delta| e^{-k T}\left[\left|\mu_{2}\right|\left(I^{\alpha-1}\left|N_{1}(t)\right|\right)\left(\eta_{2}\right)+k\left|\mu_{2}\right| \int_{0}^{\eta_{2}} e^{-k\left(\eta_{2}-s\right)}\left(I^{\alpha-1}\left|N_{1}(t)\right|\right)(s) d s\right.
$$$$
\left.+\int_{0}^{T}\left(I^{\beta-1}\left|N_{2}(t)\right|\right)(s) d s+k \int_{0}^{T} \int_{0}^{x} e^{-k(x-s)}\left(I^{\beta-1}\left|N_{2}(t)\right|\right)(s) d s d x\right]
$$$$
+|\lambda| e^{-k T}\left[\left|\rho_{2}\right|\left(I^{\beta-1}\left|N_{2}(t)\right|\right)\left(\zeta_{2}\right)+k\left|\rho_{2}\right| \int_{0}^{\zeta_{2}} e^{-k\left(\zeta_{2}-s\right)}\left(I^{\beta-1}\left|N_{2}(t)\right|\right)(s) d s\right.
$$$$
\left.+\int_{0}^{T}\left(I^{\alpha-1}\left|N_{1}(t)\right|\right)(s) d s+k \int_{0}^{T} \int_{0}^{x} e^{-k(x-s)}\left(I^{\alpha-1}\left|N_{1}(t)\right|\right)(s) d s d x\right]
$$$$
+\frac{1}{|\omega|}\left[\frac{\left|A \mu_{2}\right|}{k}\left(I^{\alpha-1}\left|N_{1}(t)\right|\right)\left(\eta_{2}\right)+\left|A \mu_{2}\right| \int_{0}^{\eta_{2}} e^{-k\left(\eta_{2}-s\right)}\left(I^{\alpha-1}\left|N_{1}(t)\right|\right)(s) d s\right.
$$$$
+\frac{|A|}{k} \int_{0}^{T}\left(I^{\beta-1}\left|N_{2}(t)\right|\right)(s) d s+\left|A-\rho_{1}\right| \int_{0}^{T} \int_{0}^{x} e^{-k(x-s)}\left(I^{\beta-1}\left|N_{2}(t)\right|\right)(s) d s d x
$$$$
+\frac{\left|B \rho_{2}\right|}{k}\left(I^{\beta-1}\left|N_{2}(t)\right|\right)\left(\zeta_{2}\right)+\left|B \rho_{2}\right| \int_{0}^{\zeta_{2}} e^{-k\left(\zeta_{2}-s\right)}\left(I^{\beta-1}\left|N_{2}(t)\right|\right)(s) d s
$$$$
+\frac{|B|}{k} \int_{0}^{T}\left(I^{\alpha-1}\left|N_{1}(t)\right|\right)(s) d s+|B-T| \int_{0}^{T} \int_{0}^{x} e^{-k(x-s)}\left(I^{\alpha-1}\left|N_{1}(t)\right|\right)(s) d s d x
$$$$
\left.+T\left|\rho_{1}\right| \int_{0}^{\zeta_{1}} e^{-k\left(\zeta_{1}-s\right)}\left(I^{\beta-1}\left|N_{2}(t)\right|\right)(s) d s+\left|\mu_{1} \rho_{1}\right| \int_{0}^{\eta_{1}} e^{-k\left(\eta_{1}-s\right)}\left(I^{\alpha-1}\left|N_{1}(t)\right|\right)(s) d s\right]
$$$$
+\int_{0}^{T} e^{-k(T-s)}\left(I^{\alpha-1}\left|N_{1}(t)\right|\right)(s) d s,
$$ 


$$
\begin{aligned}
\leq \varepsilon_{1}\left[|\Delta| e^{-k T} \mu_{2}\right. & \left(\left(I^{\alpha-1} 1\right)\left(\eta_{2}\right)+k \int_{0}^{\eta_{2}} e^{-k\left(\eta_{2}-s\right)}\left(I^{\alpha-1} 1\right)(s) d s\right) \\
& +|\lambda| e^{-k T}\left(\int_{0}^{T}\left(I^{\alpha-1} 1\right)(s) d s+k \int_{0}^{T} \int_{0}^{x} e^{-k(x-s)}\left(I^{\alpha-1} 1\right)(s) d s d x\right) \\
& +\frac{1}{|\omega|}\left(\frac{\left|A \mu_{2}\right|}{k}\left(I^{\alpha-1} 1\right)\left(\eta_{2}\right)+\left|A \mu_{2}\right| \int_{0}^{\eta_{2}} e^{-k\left(\eta_{2}-s\right)}\left(I^{\alpha-1} 1\right)(s) d s+\frac{|B|}{k} \int_{0}^{T}\left(I^{\alpha-1} 1\right)(s) d s\right. \\
& \left.+|B-T| \int_{0}^{T} \int_{0}^{x} e^{-k(x-s)}\left(I^{\alpha-1} 1\right)(s) d s d x+\left|\mu_{1} \rho_{1}\right| \int_{0}^{\eta_{1}} e^{-k\left(\eta_{1}-s\right)}\left(I^{\alpha-1} 1\right)(s) d s\right) \\
& \left.+\int_{0}^{T} e^{-k(T-s)}\left(I^{\alpha-1} 1\right)(s) d s\right] \\
& +\varepsilon_{2}\left[|\Delta| e^{-k T}\left(\int_{0}^{T}\left(I^{\beta-1} 1\right)(s) d s+k \int_{0}^{T} \int_{0}^{x} e^{-k(x-s)}\left(I^{\beta-1} 1\right)(s) d s d x\right)\right. \\
& +|\lambda| e^{-k T}\left(\left|\rho_{2}\right|\left(I^{\beta-1} 1\right)\left(\zeta_{2}\right)+k\left|\rho_{2}\right| \int_{0}^{\zeta_{2}} e^{-k\left(\zeta_{2}-s\right)}\left(I^{\beta-1} 1\right)(s) d s\right) \\
& +\frac{1}{|\omega|}\left(\frac{|A|}{k} \int_{0}^{T}\left(I^{\beta-1} 1\right)(s) d s+\left|A-\rho_{1}\right| \int_{0}^{T} \int_{0}^{x} e^{-k(x-s)}\left(I^{\beta-1} 1\right)(s) d s d x+\frac{\left|B \rho_{2}\right|}{k}\left(I^{\beta-1} 1\right)\left(\zeta_{2}\right)\right. \\
& \left.\left.+\left|B \rho_{2}\right| \int_{0}^{\zeta_{2}} e^{-k\left(\zeta_{2}-s\right)}\left(I^{\beta-1} 1\right)(s) d s\right)\right]=\varepsilon_{1} M_{1}+\varepsilon_{2} M_{2} .
\end{aligned}
$$

By the same method, we can obtain that:

$$
\left|y(t)-y^{*}(t)\right| \leq M_{3} \varepsilon_{1}+M_{4} \varepsilon_{2},
$$

where $M_{\mathrm{i}}, \mathrm{i}=1,2,3,4$ are mentioned before. By (20) and (21), the nonlinear sequential coupled system of Caputo fractional differential equations is Ulam-Hyers stable and consequently, the system (1) is Ulam-Hyers stable.

Example 1. Consider the following system of a fractional differential equation:

$$
\left\{\begin{array}{c}
{ }^{\mathrm{c}} D^{1 / 2}(D+1) x(t)=\frac{1}{8 \pi \sqrt{49+t^{2}}}\left(\frac{|x(t)|}{1+|x(t)|}+\sin y(t)\right)+\frac{1}{4}, t \in[0,1] \\
{ }^{\mathrm{c}} D^{1 / 3}(D+1) y(t)=\frac{1}{2 \pi(4+t)^{2}}\left(\sin (x(t))+\frac{y(t)}{1+|x(t)|}\right)+1, t \in[0,1] \\
\int_{0}^{1} x(s) d s=y(1 / 2), \int_{0}^{1} x^{\prime}(s) d s=-2 y^{\prime}(1 / 2), \\
\int_{0}^{1} y(s) d s=-3 x(1 / 3), \int_{0}^{1} y^{\prime}(s) d s=x^{\prime}(1) .
\end{array}\right.
$$

Here:

$$
k=1, \alpha=\frac{3}{2}, \beta=\frac{4}{3}, T=1, \rho_{1}=1, \zeta_{1}=\frac{1}{2}, \rho_{2}=-2, \zeta_{2}=\frac{1}{2}, \mu_{1}=-3, \eta_{1}=\frac{1}{3}, \mu_{2}=1, \eta_{2}=1 .
$$

We found:

$$
M_{1}=4.5398, M_{2}=4.9766, M_{3}=2.7046, M_{4}=5.872, h_{1}=\frac{1}{56 \pi}, h_{2}=\frac{1}{32 \pi} .
$$

It's clear that $f, g$ are jointly continuous functions, where:

$$
\begin{aligned}
& f(t, x, y)=\frac{1}{8 \pi \sqrt{49+t^{2}}}\left(\frac{|x(t)|}{1+|x(t)|}+\sin y(t)\right)+\frac{1}{4}, \\
& g(t, x, y)=\frac{1}{2 \pi(4+t)^{2}}\left(\sin (x(t))+\frac{y(t)}{1+|x(t)|}\right)+1 .
\end{aligned}
$$


Now, check that $h_{1}\left(M_{1}+M_{3}\right)+h_{2}\left(M_{2}+M_{4}\right)<1$.

Hence:

$$
\frac{1}{56 \pi}(7.2444)+\frac{1}{32 \pi}(10.8486)=0.149<1 .
$$

Thus, all the conditions of Theorem 1 are satisfied, and problem (22) has a unique solution on $[0,1]$.

Example 2. Consider the following system of fractional differential equation:

$$
\left\{\begin{array}{c}
{ }^{\mathrm{c}} D^{\frac{1}{2}}(D+1) x(t)=\frac{1}{60+t^{2}}+\frac{e^{-t} \cos (x(t))}{2 \sqrt{6400+t^{4}}}+\frac{|y(t)|}{140\left(1+x^{2}(t)\right)} t \in[0,1] \\
{ }^{\mathrm{c}} D^{\frac{1}{3}}(D+1) y(t)=\frac{1}{\sqrt{25+t^{2}}} \cos ^{2}(t)+\frac{e^{-t} \sin (x(t))}{130}+\frac{y(t)}{120}, t \in[0,1] \\
\int_{0}^{1} x(s) d s=y(1 / 2), \int_{0}^{1} x^{\prime}(s) d s=-2 y^{\prime}(1 / 2), \\
\int_{0}^{1} y(s) d s=-3 x(1 / 3), \int_{0}^{1} y^{\prime}(s) d s=x^{\prime}(1) .
\end{array}\right.
$$

Here:

$$
k=1, \alpha=\frac{3}{2}, \beta=\frac{4}{3}, T=1, \rho_{1}=1, \zeta_{1}=\frac{1}{2}, \rho_{2}=-2, \zeta_{2}=\frac{1}{2}, \mu_{1}=-3, \eta_{1}=\frac{1}{3}, \mu_{2}=1, \eta_{2}=1 .
$$

We found:

$$
M_{1}=4.5398, M_{2}=4.9766, M_{3}=2.7046, M_{4}=5.872 .
$$

It's clear that $\mathrm{f}, \mathrm{g}$ are jointly continuous functions and:

$$
\begin{aligned}
|f(t, x, y)| & \leq \frac{1}{60}+\frac{1}{160}|x|+\frac{1}{140}|y|, \\
|g(t, x, y)| & \leq \frac{1}{5}+\frac{1}{130}|x|+\frac{1}{120}|y| .
\end{aligned}
$$

Thus: $\theta_{0}=\frac{1}{60}, \theta_{1}=\frac{1}{160}, \theta_{2}=\frac{1}{140}, \vartheta_{0}=\frac{1}{5}, \vartheta_{1}=\frac{1}{130}, \vartheta_{2}=\frac{1}{120}$.

Note that:

$$
\left(M_{1}+M_{3}\right) \theta_{1}+\left(M_{2}+M_{4}\right) \vartheta_{1}=0.1288<1,
$$

and:

$$
\left(M_{1}+M_{3}\right) \theta_{2}+\left(M_{2}+M_{4}\right) \vartheta_{2}=0.1422<1 .
$$

Thus, all the conditions of Theorem 2 are satisfied, and problem (23) has at least one solution on $[0,1]$.

\section{Conclusions}

The existence, stability, and uniqueness for the solution of the coupled system of Caputo-type sequential fractional differential equations that involve integral boundary conditions were investigated. Leray-Schauder's alternative was implemented to show the existence of the proposed system and the Banach's contraction principle was used to examine the uniqueness of the solution. The Ulam-Hyers stability of the proposed system was investigated, and it was found that the presented system was stable and unique; an example has been given to illustrate certain related aspects. The presented approach may be extended to obtain numerical solutions for a coupled system of Caputo-type sequential fractional differential equations, which will be discussed in detail at a later stage. 
Author Contributions: Writing—original draft, A.A.-k.; Methodology, A.A.-k. software, H.Z.; validation, O.A.; formal analysis, A.A.-k.; investigation, S.B.; writing-original draft preparation, H.Z.; writing-review and editing, A.A.-k., and H.Z.; visualization, O.A. Funding acquisition, S.B. All authors have read and agreed to the published version of the manuscript.

Funding: This research received no external funding.

Institutional Review Board Statement: Not applicable.

Informed Consent Statement: Not applicable.

Data Availability Statement: Not applicable.

Acknowledgments: The authors wish to thank the anonymous reviewers for their valuable comments and suggestions.

Conflicts of Interest: The authors declare no conflict of interest.

\section{References}

1. Podlubny, I. Fractional Differential Equations; Academic Press: San Diego, CA, USA, 1999.

2. Kilbas, A.A.; Srivastava, H.M.; Trujillo, J.J. Theory and Applications of Fractional Differential Equations; North-Holland Mathematics Studies; Elsevier Science B.V.: Amsterdam, The Netherlands, 2006; Volume 204.

3. Miller, K.S.; Ross, B. An Introduction to the Fractional Calculus and Fractional Differential Equations; Wiley and Sons: New York, NY, USA, 1993.

4. Sabatier, J.; Agrawal, O.; Machado, J. (Eds.) Advances in Fractional Calculus: Theoretical Developments and Applications in Physics and Engineering; Springer: Dordrecht, The Netherlands, 2007.

5. Ahmad, B.; Sivasundaram, S. On four-point nonlocal boundary value problems of nonlinear integro-differential equations of fractional order. Appl. Math. Comput. 2010, 217, 480-487. [CrossRef]

6. Gambo, Y.; Jarad, F.; Baleanu, D.; Abdeljawad, T. On caputo modification of the Hadamard fractional derivatives. Adv. Differ. Equ. 2014, 2014, 780636. [CrossRef]

7. Herzallah, M.; Baleanu, D. On fractional order hybrid differential equations. Abstr. Appl. Anal. 2014, 2014, 389386. [CrossRef]

8. Gomez, A.; Jose, F.; Baleanu, D. Solutions of the telegraph equations using a fractional calculus approach. Proc. Rom. Acad. Ser. A Math. Phys. Tech. Sci. Inf. Sci. 2014, 15, 27-34.

9. Malkawi, E.; Baleanu, D. Fractional killing-yano tensors and killing vectors using the caputo derivative in some one-and two-dimensional curved space. Abstr. Appl. Anal. 2014, 2014, 290694. [CrossRef]

10. Ahmad, B.; Nieto, J.J.; Pimentel, J. Some boundary value problems of fractional differential equations and inclusions. Comput. Math. Appl. 2011, 62, 1238-1250. [CrossRef]

11. Ahmad, B.; Ntouyas, S.K.; Alsaedi, A. New existence results for nonlinear fractional differential equations with three-point integral boundary conditions. Adv. Differ. Equ. 2011, 2011, 107384. [CrossRef]

12. Ahmad, B.; Ntouyas, S.K. A four-point nonlocal integral boundary value problem for fractional differential equations of arbitrary order. Electron. J. Qual. Theory Differ. Equ. 2011, 22, 1-15. [CrossRef]

13. Baleanu, D.; Mustafa, O.G.; Agarwal, R.P. On lp-solutions for a class of sequential fractional differential equations. Appl. Math. Comput. 2011, 218, 2074-2081. [CrossRef]

14. Wang, G.; Ahmad, B.; Zhang, L.; Agarwal, R.P. Nonlinear fractional integro-differential equations on unbounded domains in a banach space. J. Comput. Appl. Math. 2013, 249, 51-56.

15. Graef, J.R.; Kong, L.; Wang, M. Existence and uniqueness of solutions for a fractional boundary value problem on a graph. Fract. Calc. Appl. Anal. 2014, 17, 499-510. [CrossRef]

16. Liu, X.; Liu, Z.; Fu, X. Relaxation in nonconvex optimal control problems described by fractional differential equations. J. Math. Anal. Appl. 2014, 409, 446-458. [CrossRef]

17. Zhai, C.; Xu, L. Properties of positive solutions to a class of four-point boundary value problem of caputo fractional differential equations with a parameter. Commun. Nonlinear Sci. Numer. Simul. 2014, 19, 2820-2827. [CrossRef]

18. Granas, A.; Dugundji, J. Fixed Point Theory; Springer: New York, NY, USA, 2005.

19. Boutiara, A.; Etemad, S.; Alzabut, J.; Hussain, A.; Subramanian, M.; Rezapour, S. On a nonlinear sequential four-point fractional q-difference equation involving q-integral operators in boundary conditions along with stability criteria. Adv. Differ. Equ. 2021, 2021, 367. [CrossRef]

20. Ahmad, B.; Nieto, J.J. Boundary value problems for a class of sequential integrodifferential equations of fractional order. J. Funct. Spaces Appl. 2013, 2013, 149659. [CrossRef]

21. Mahmudov, N.I.; Bawaneh, S.; Al-Khateeb, A. On a coupled system of fractional differential equations with four point integral boundary conditions. Mathematics 2019, 7, 279. [CrossRef]

22. Mahmudov, N.; Al-Khateeb, A. Existence and Stability Results on Hadamard Type Fractional Time-Delay Semilinear Differential Equations. Mathematics 2020, 8, 1242. [CrossRef] 
23. Mahmudov, N.I.; Al-Khateeb, A. Existence and Ulam-Hyers stability of coupled sequential fractional differential equations with integral boundary conditions. J. Inequalities Appl. 2019, 2019, 165. [CrossRef]

24. Muthaiah, S.; Baleanu, D.; Thangaraj, N.G. Existence and Hyers-Ulam type stability results for nonlinear coupled system of Caputo-Hadamard type fractional differential equations. Aims Math. 2021, 6, 168-194. [CrossRef]

25. Frassu, S.; van der Mee, C.; Viglialoro, G. Boundedness in a nonlinear attraction-repulsion Keller-Segel system with production and consumption. J. Math. Anal. Appl. 2021, 504, 125428. [CrossRef]

26. Li, T.; Viglialoro, G. Boundedness for a nonlocal reaction chemotaxis model even in the attraction-dominated regime. Differ. Integral Equ. 2021, 34, 315-336.

27. Mahmudov, N.I.; Al-Khateeb, A. Stability, existence and uniqueness of boundary value problems for a coupled system of fractional differential equations. Mathematics 2019, 7, 354. [CrossRef]

28. Ahmad, B.; Ntouyas, S.K. Existence results for a coupled system of Caputo type sequential fractional differential equations with nonlocal integral boundary conditions. Appl. Math. Comput. 2015, 266, 615-622. [CrossRef] 